\title{
Headspace (HS) et micro-extraction en phase solide (SPME). Théorie et applications
}

\section{Headspace (HS) and solid phase micro-extraction (SPME). Theory and applications}

\author{
Yves Papet ${ }^{\star}$, Bertrand Brunet, Patrick Mura \\ Centre Hospitalier Universitaire, Service de toxicologie et pharmacocinétique, BP 577, 86021 Poitiers Cedex, France
}

\begin{abstract}
Résumé - Les techniques headspace et la micro-extraction en phase solide sont individuellement des modes de préparation d'échantillon très utilisés dans de nombreux domaines industriels et en biologie. Leur association permet d'augmenter la sensibilité des analytes recherchés et quantifiés et ainsi d'abaisser les limites de quantification et de détection. La technique headspace ou espace de tête est basée sur la volatilité de l'analyte recherché dans une matrice complexe non chromatographiable. Elle peut prendre deux aspects, soit statique, où le prélèvement se fait dans l'espace de tête directement avec un volume constant, soit dynamique après piégeage de l'analyte sur un support que l'on désorbe par un choc thermique. La micro-extraction permet de piéger des analytes avec une aiguille rétractable recouverte d'une phase adaptée aux analytes dans un milieu ambiant ou confiné (par exemple un espace de tête). Cette aiguille est introduite à l'intérieur d'un injecteur et les analytes sont désorbés thermiquement ou élués en fonction de la technique analytique utilisée. Deux applications sont présentées : le dosage des alcools dans le sang total et le suivi des esters d'éthyle dans le whisky. La diversité de ces deux exemples illustre bien l'écclectisme de ces deux types de préparation d'échantillon. Il s'agit donc de deux méthodes de préparation simples et rapides qui permettent de doser les substances volatiles. Associées, elles permettent d'augmenter la sensibilité et donc d'abaisser les limites de quantification et de détection.
\end{abstract}

Mots clés : Headspace, micro-extraction, alcools, esters d'éthyle

\begin{abstract}
Headspace and solid phase micro-extraction are two methods of sample preparation widely used in industrial fields and biology. Their combination helps to increase the sensitivity of analytes and consequently to obtain lower limits of quantification and detection. The headspace technique is based on the volatility of the analyte present in a complex matrix. It can take two forms, either static where the volatil analyte is sampled directly in the headspace with a constant volume or dynamic after trapping the analyte on a support which is desorbed by thermic shock. The microextraction allows trapping of analytes with a retractable needle covered with a suitable phase in a confined environment (such as headspace). The needle is inserted inside an injector and the analytes are thermally desorbed or eluted according to the analytical technique used. Two applications are presented: the determination of various alcohols in whole blood and the monitoring of ethyl esters in whiskey. The diversity of these two examples illustrates the eclecticism of these two types of sample preparation. Both preparation methods allow simple and rapid assay of volatile substances. Combined, they can increase the sensitivity and therefore lower limits of quantification and detection are observed.
\end{abstract}

Key words: Headspace, micro-extraction, alcohols, ethyl ester

Reçu le 10 mars 2010, accepté après modifications le 3 mai 2010 Publication en ligne le 4 août 2010

\section{Introduction}

L'headspace ou espace de tête est une technique très utilisée dans de nombreux domaines pour analyser les substances volatiles dans des matrices complexes non chromatographiables. Le mode analytique utilisé pour ces substances est

\footnotetext{
^Correspondance : Yves Papet, y.papet@chu-poitiers.fr
}

la chromatographie en phase gazeuse couplée soit à un détecteur à ionisation de flamme soit à un spectromètre de masse en impact électronique ou ionisation chimique.

La micro-extraction en phase solide fut proposée par Pawliszyn [1] et développée par la société Supelco. La phase solide est déposée sur un support rétractable après exposition aux analytes. L'analyse est effectuée soit en chromatogaphie en phase gazeuse par désorption thermique de l'aiguille dans 


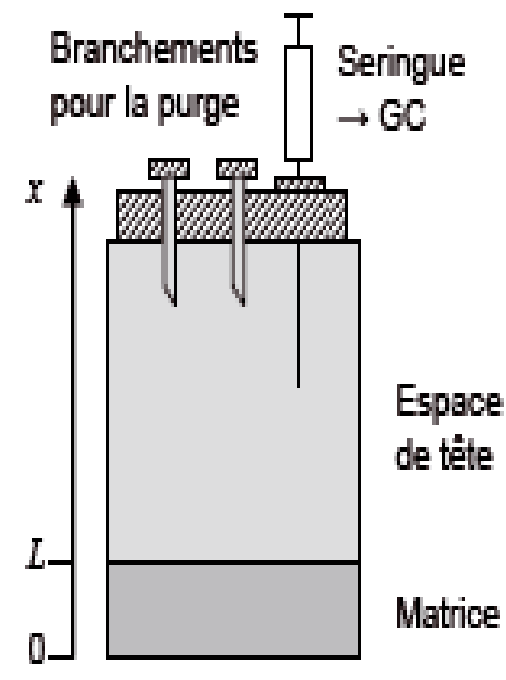

Fig. 1. Schéma du système espace de tête ou headspace.

l'injecteur soit par élution dans le cadre de la chromatographie liquide. Quel que soit le type de chromatographie utilisée, il faudra déterminer le meilleur détecteur possible pour l'application envisagée.

En espace de tête, seules les substances volatiles pourront être détectées, ce qui permet de s'affranchir des matrices, qui peuvent être très différentes les unes des autres.

En micro-extraction en phase solide, soit le prélèvement est effectué dans l'espace de tête et on se retrouve dans la configuration précédente, soit le prélèvement est effectué dans la matrice qui est souvent un liquide et la sélection des molécules d'intérêt est réalisée en utilisant la sélectivité des différentes phases stationnaires du marché.

\section{Méthode}

L'headspace ou espace de tête est la partie supérieure du flacon qui est comprise entre la matrice et le bouchon assurant l'étanchéité du flacon (voir figure 1). Les molécules volatiles se retrouvent dans cet espace et sont celles qui sont à identifier et à quantifier.

L'équilibre liquide /gaz dans cet espace de tête est régi par un coefficient :

$$
\mathrm{Kh}=\mathrm{C}_{\mathrm{L}} / \mathrm{C}_{\mathrm{G}} \text {. }
$$

C est la concentration de l'analyte soit dans le liquide si la matrice est un liquide soit dans le gaz. Cet équilibre peut être déplacé en modifiant certains paramètres :

- la température : quand la température du flacon est augmentée, le passage à l'état de vapeur des analytes est favorisé ;

- l'agitation, qui facilite aussi le passage des analytes dans l'espace de tête ;

- le temps de chauffage, qui permet aussi de jouer sur le passage des molécules dans l'espace de tête.

Enfin, il est possible de faire varier le $\mathrm{pH}$ et «l'effet de sel » de manière à modifier l'équilibre liquide/gaz et avoir ainsi plus de substances volatiles dans la phase gazeuse.

\section{Étape d'extraction}

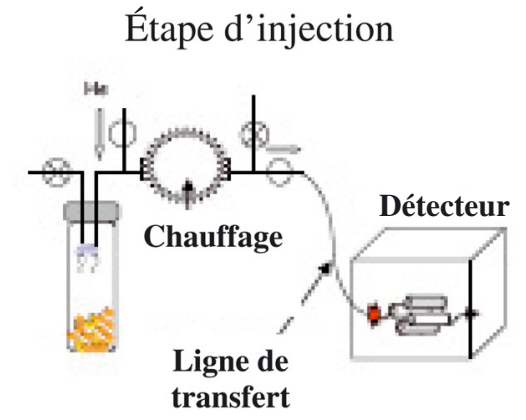

Fig. 2. Principe de l'headspace ou espace de tête en mode dynamique.

Lorsque les meilleures conditions de température, de temps de chauffage et de $\mathrm{pH}$ ont été déterminées, il est possible de commencer l'analyse selon deux modes différents soit :

\section{En mode statique}

On prélève après une période d'équilibrage thermodynamique dans l'espace de tête un volume fixe de manière manuelle ou automatique qui est injecté dans le chromatographe. Dans ces conditions, la quantité de chaque composé volatil contenue dans l'espace de tête est proportionnelle à la concentration contenue dans la matrice. Après étalonnage de préférence avec un étalon interne, il est possible de déterminer les concentrations dans la matrice, qui sont équivalentes à celles de l'espace de tête.

\section{En mode dynamique}

Au lieu d'opérer en vase clos, les substances volatiles sont entraînées par un gaz vecteur (par exemple l'hélium) soit dans l'espace de tête soit en le faisant directement «barboter» dans la matrice. Les substances volatiles sont adsorbées sur un piège qui les concentre. Ensuite il est procédé à une désorption thermique du piège pour injecter ces substances piégées dans le chromatographe (figure 2).

La microextraction en phase solide (SPME) développée par la société Supelco et reprise par de nombreux constructeurs est une technique qui s'inspire et de l'extraction sur colonne et de l'espace de tête. Le principe de fonctionnement est illustré par la figure 3 .

Le dispositif comprend une seringue munie d'une aiguille creuse dans laquelle peut coulisser un piston munie d'une courte fibre. Cette fibre en silice imprégnée d'une phase stationnaire est introduite dans le liquide ou dans l'espace situé au-dessus de la surface. Elle concentre les composés extractibles de la matrice. La fibre est ensuite insérée dans l'injecteur par l'intermédiaire de la seringue et de son piston rétractable. Elle subit une désorption thermique dans la colonne. La même fibre peut servir une cinquantaine de fois.

Il existe de nombreuses possibilités de phases stationnaires :

- Le PDMS polydiméthyl siloxane, utilisé pour les molécules non polaires de masses moléculaires comprises entre 125 et 600 . 


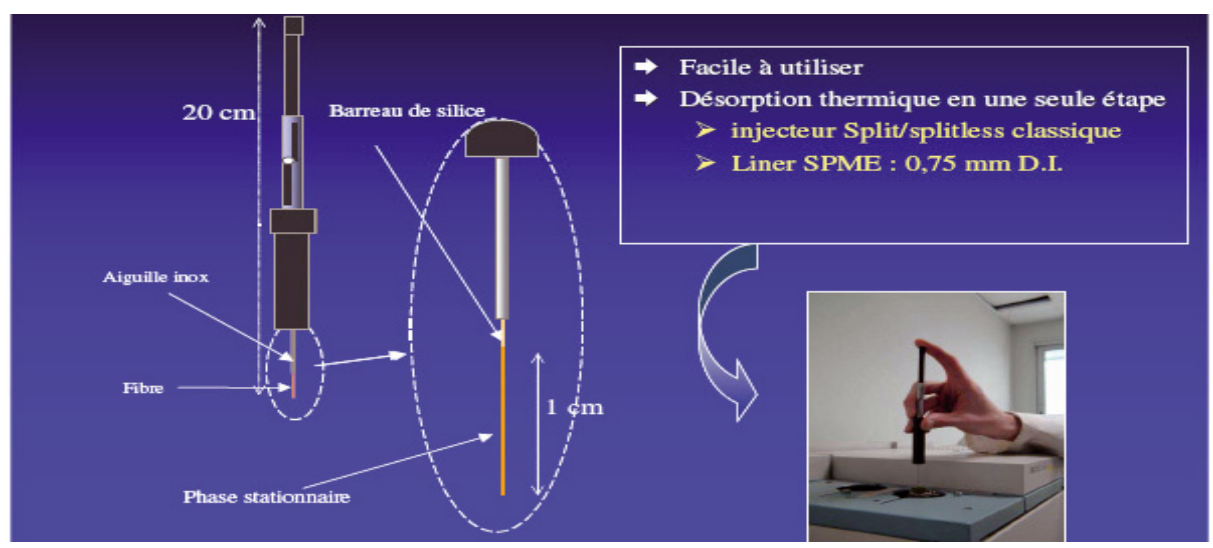

Fig. 3. Principe de la microextraction en phase solide (SPME).

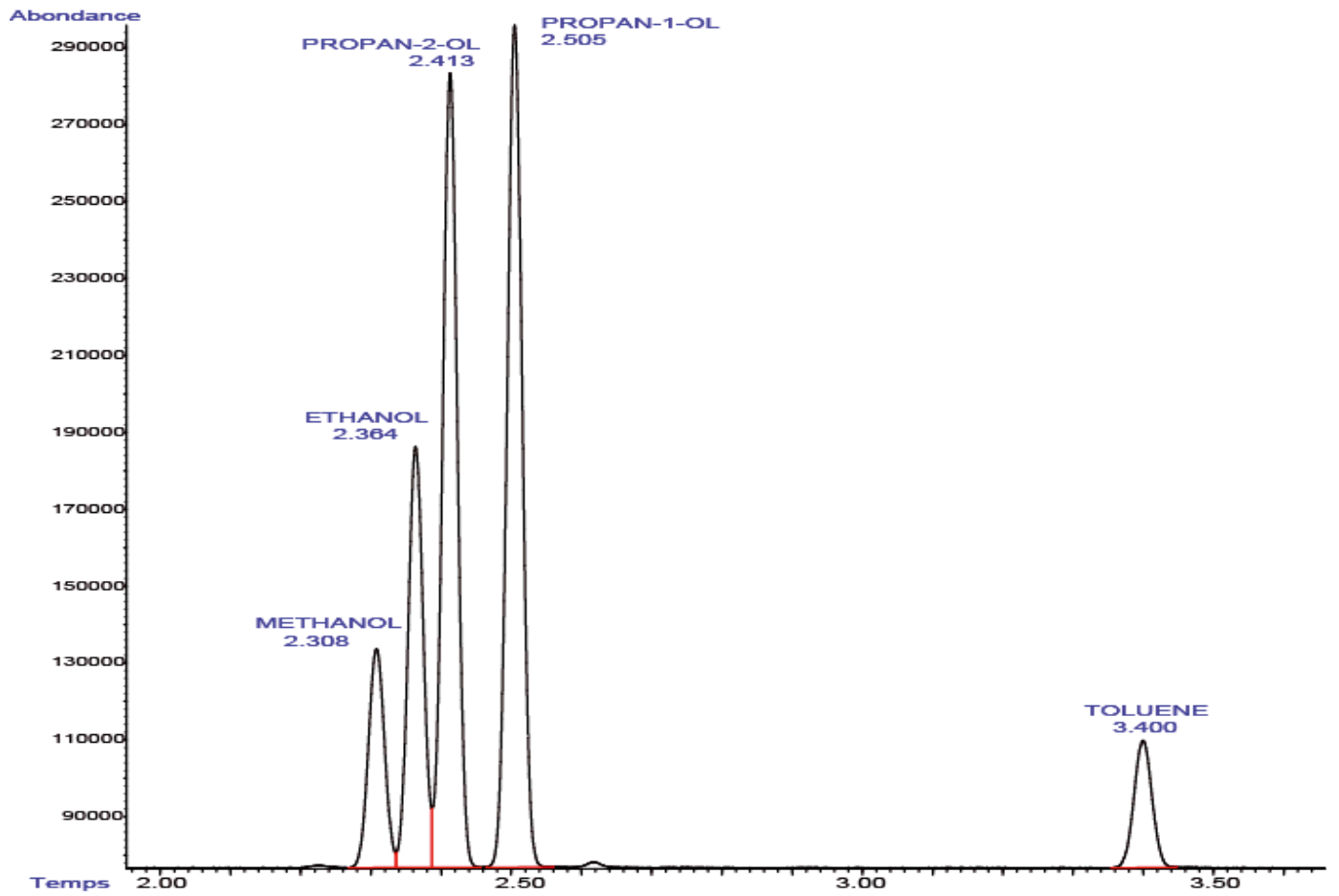

Fig. 4. Chromatogramme d'un mélange d'alcools et de toluène (HS-GC-MS).

- Le polyacrylate, utilisé pour les molécules polaires semivolatiles ayant une masse moléculaire de 80 à 300.

- Le PDMS/DVB (divinylbenzène), utilisé pour les amines et les composés nitro-aromatiques.

- Le DVB/carboxen/PDMS, utilisé pour les composés volatiles et semi volatiles de $\mathrm{C} 3$ à $\mathrm{C} 20$.

- Le carboxen/PDMS, utilisé pour les composés à faible poids moléculaire de 30 à 225.

\section{Les applications}

Les applications de l'headspace couplée ou non à la SPME sont aussi nombreuses que variées. Les deux applications que nous présentons ici sont le dosage des alcools et du toluène dans une matrice biologique « le sang total» et le dosage des esters d'ethyle dans le whisky.

\subsection{Le dosage des alcools dans le sang total}

Dans un flacon, sont introduits $200 \mu \mathrm{L}$ de sang total et $200 \mu \mathrm{L}$ d'étalon interne. Le mélange est ensuite chauffé pendant $6 \mathrm{~min}$ à $80^{\circ} \mathrm{C}$ et $1 \mathrm{~mL}$ est alors injecté en mode statique.

La colonne utilisée est un OPTIMA 1 de $30 \mathrm{~m}$ de longueur et de $0,32 \mathrm{~mm}$ de diamètre et l'épaisseur du film de phase est de $1 \mu \mathrm{m}$. Le détecteur est soit à ionisation de flamme s'il s'agit de molécules identifiées, soit un spectromètre de masse pour des molécules non identifiées.

La figure 4 illustre l'exemple d'un chromatogramme obtenu en GC-MS avec injection en espace de tête lors de l'analyse d'un mélange d'alcools et de toluène. 


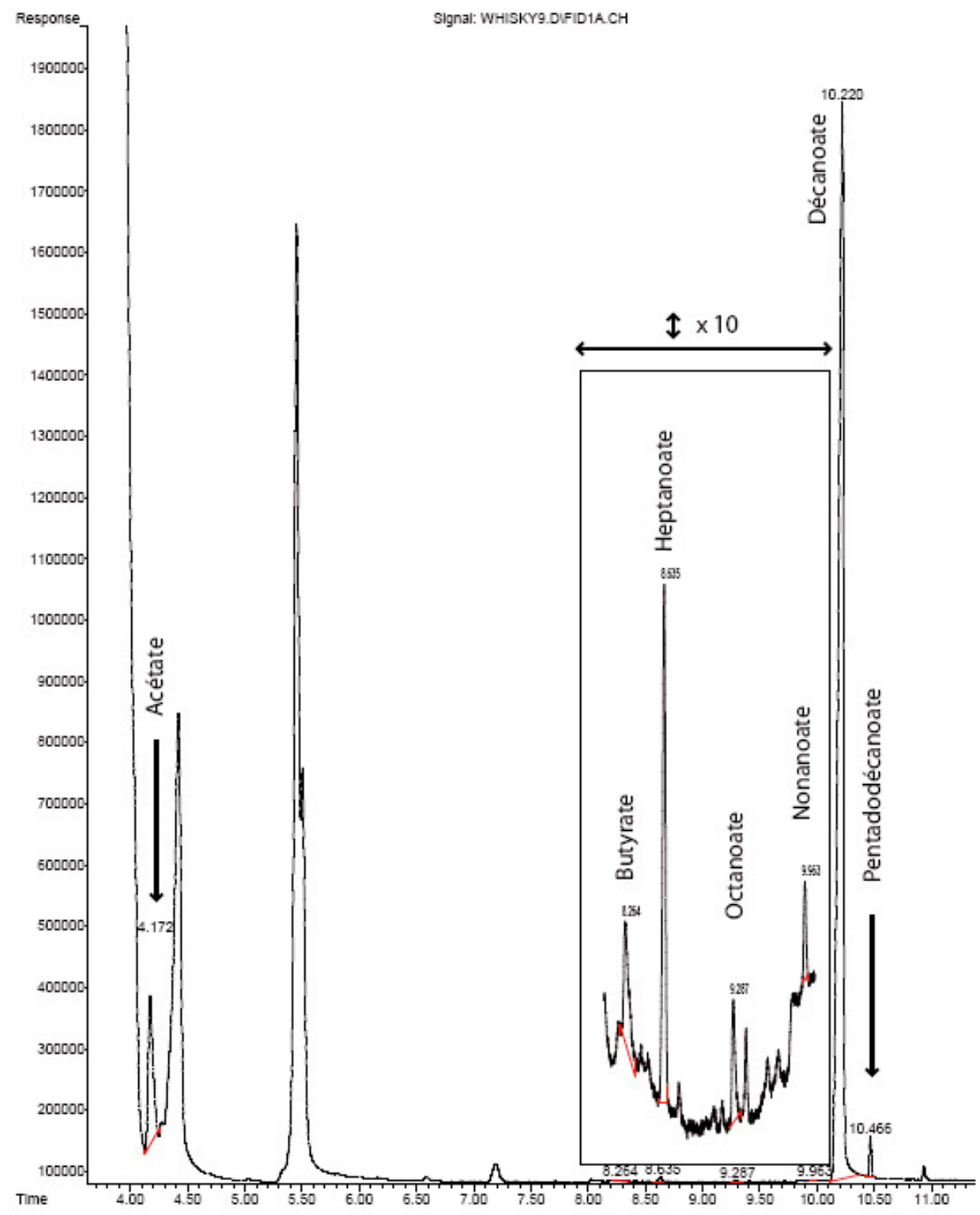

Fig. 5. Chromatogramme des esters d'éthyle d'un échantillon de whisky.

\subsection{Le dosage des esters d'éthyle dans le whisky}

Cette étude pour amateurs de whisky est un résumé des articles de Conner et coll. parus en 1998 et 1999 [2,3]. Ces auteurs ont étudié entre autres le devenir des esters d'éthyle en fonction de la concentration en alcool. Les esters résultent de la réaction d'un acide et d'un alcool. La nomenclature veut que le premier terme provienne de l'acide et se termine en « oate» et le deuxième terme provienne de l'alcool et se termine en «yle».

Les arômes développés par le whisky sont essentiellement dus aux esters d'éthyle. Les esters d'éthyle à chaîne carbonée longue sont responsables des goûts et arômes désagréables alors que les esters d'éthyle à chaîne carbonée courte sont responsables des arômes agréables comme les parfums de fruit d'ananas etc. Pour ce faire, ils ont dosé les concentrations d'esters d'éthyle suivant : l'acétate, le butyrate, l'hexanoate, l'octanoate, le décanoate et le dodécanoate contenus dans l'espace de tête en fonction des concentrations en alcool. Les concentrations dans l'espace de tête sont exprimées en coefficient d'activité. La figure 5 montre un chromatogramme des esters d'éthyle obtenu dans notre laboratoire à partir d'un échantillon de whisky.

Lorsque la concentration en alcool diminue, le coefficient d'activité croît, ce qui correspond à une diminution des esters d'éthyle.

Cette diminution des esters d'éthyle est d'autant plus marquée que la chaîne carbonée est courte. Si l'on rapporte cette interprétation au whisky, on constate que lorsque la concentration d'alcool dans le whisky passe de $40 \%$ à $35 \%$ les esters d'éthyle les plus volatiles, donc responsables des goûts et arômes agréables, sont les premiers à envahir l'espace de tête. Cela signifie aussi que lors de l'ouverture de la bouteille ils seront les premiers à disparaître au dépend du whisky restant. 
Au fur et à mesure de la consommation, le degré d'alcool diminue, les esters d'éthyle les plus légers et responsables de la qualité diminuent et les plus lourds, responsables des goûts et arômes désagréables augmentent. Pour remédier à cette conséquence désagréable, il y à deux manières de procéder. La première consiste à mettre le whisky dans un flacon approprié après chaque consommation de manière à réduire l'espace de tête au maximum. La seconde solution est d'augmenter le degré alcoolique du whisky de manière à diminuer le coefficient d'activité des esters d'éthyle. Cette solution n'a pas été retenue car les taxes sur l'alcool au Royaume-Uni sont très élevées et devraient être répercutées sur le prix de vente.

\section{Conclusion}

Espace de tête (headspace) et SPME sont deux techniques d'extraction simples et rapides. Associées, elles permettent d'augmenter la sensibilité et d'abaisser les limites de détection et de quantification. Elles sont utilisées pour doser des substances volatiles dans de nombreux domaines comme la biologie, l'œnologie, la cosmétologie, l'étude des essences essentielles des plantes etc...

Conflit d'intérêt. Les auteurs déclarent ne pas avoir de conflit d'intérêt.

\section{Références}

1. Pawliszyn J. Theory of solid-phase microextraction. J Chromatogr Sci. 2000; 38(7): 270-278.

2. Conner JM, Birkmyre L, Paterson A, Piggott JR. Headspace concentrations of ethyl esters at different alcoholic strengths. J Sci Food Agr. 1998; 77: 121-126.

3. Conner JM, Paterson A, Piggott JR. Release of distillate flavour compounds in Scotch malt whisky. J Sci Food Agr. 1999; 79: 1015-1020. 